\title{
Numerical Solution and Stability Analysis of Huxley Equation
}

Saad A. Manaa

saad.manaa@uoz.edu.kr
Mohammad Sabawi

mohammad.sabawi@tu.edu.iq

\section{College of Computers Sciences and Mathematics}

University of Mosul, Iraq

\section{Received on: $25 / 01 / 2005$}

Accepted on: 05/04/2005

\section{ABSTRACT}

The numerical solution of Huxley equation by the use of two finite difference methods is done. The first one is the explicit scheme and the second one is the Crank-Nicholson scheme. The comparison between the two methods showed that the explicit scheme is easier and has faster convergence while the Crank-Nicholson scheme is more accurate. In addition, the stability analysis using Fourier (von Neumann) method of two schemes is investigated. The resulting analysis showed that the first scheme is conditionally stable if $r \leq \frac{2-a \beta \Delta t}{4}, \Delta t \leq \frac{2(\Delta x)^{2}}{4-a \beta(\Delta x)^{2}}$ and the second scheme is unconditionally stable.

Keywords: Finite Difference Methods, Explicit Scheme, Crank-Nicholson, Huxley Equation, Stability Analysis.

$$
\begin{aligned}
& \text { Huxley الحل العدي وتحليل الاستقرارية لمعادئة }
\end{aligned}
$$

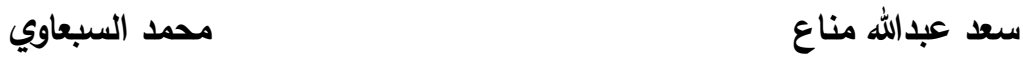

$$
\begin{aligned}
& \text { كلية علوم الحاسبات والرياضيات، جامعة الدوصل }
\end{aligned}
$$

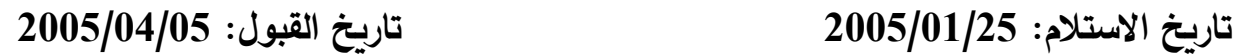

$$
\begin{aligned}
& \text { الملخص } \\
& \text { تم حل معادلة Huxley باستخدام طريقتين من طرائق الفرو قات المنتهية: الأولى هي } \\
& \text { الطريقـة الصـريحة والثانيـة هي طريقـة Crank-Nicholson إذ تم عمل مقارنـة بين نتائج كلتـا } \\
& \text { الطريقتين وقد تبين إن الطريقة الأولى هي الأسهل والأسرع تقارباً في حين كانت الطريقة الثانية هي } \\
& \text { من } r \leq \frac{2-a \beta \Delta t}{4}, \Delta t \leq \frac{2(\Delta x)^{2}}{4-a \beta(\Delta x)^{2}} \\
& \text { مشروط. }
\end{aligned}
$$

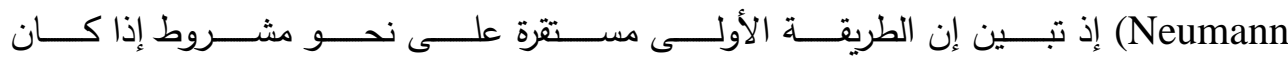




\section{الكلمات المفتاحية: طريقة الفروقات المنتهية، الطريقة الصريحة، تحليل الاستقرارية}

\section{Introduction}

It is probably not an overstatement to say that almost all partial differential equations (PDEs) that arise in a practical setting are solved numerically on a computer. Since the development of high-speed computing devices, the numerical solution of PDEs has been in active state with the invention of new algorithms and the examination of the underlying theory.

This is one of the most active areas in applied mathematics and it has a great impact on science and engineering because of the ease and efficiency it has shown in solving even the most complicated problems. The basic idea of the method of finite differences is to cast the continuous problem described by the PDE and auxiliary conditions into a discrete problem that can be solved by a computer in finitely many steps. The discretization is accomplished by restricting the problem to a set of discrete points. By systematic procedure, we then calculate the unknown function at those discrete points. Consequently, a finite difference technique yields a solution only at discrete points in the domain of interest rather than, as we expect for an analytical calculation, a formula or closed-form solution valid at all points of the domain [11]. Manoranjan et al [12] obtained estimates for the critical lengths of the domain at which bifurcation occurs in the cases $b=0, a, 0<a \leq 1 / 2$, and 1 .

Manoranjan [13] studied in detail the solutions bifurcating from the equilibrium state $u=a$. Eilbeck and Manoranjan [3] considered different types of basis functions for the pseudo-spectral method applied to the nonlinear reaction-diffusion equation in 1- and 2- space dimensions. Eilbeck [4] extended the pseudo-spectral method to follow steady state solutions as a function of the problem parameter, using path-following techniques. Fath and Domanski [6] studied the cellular differentiation in a developing organism via a discrete bistable reaction-diffusion model and they used the numerical simulation to support their expectations of the qualitative behavior of the system. Lewis and Keener [10] studied the propagation failure using the one -dimensional scalar bistable equation by a passive gap and they used the numerical simulation in their study. Binczak et al [1] compared the numerical predictions of the simple myelinated nerve fibers with the theoretical results in the continuum and discrete limits. Broadbridge et al [2] re-examined the derivation of the gene- transport equations and used the Gaussian clump of alleles by the use of a numerical method-oflines by using PDETWO program. Lefantzi et al [9] presented their findings for various orders of spatial discretizations as applied to SAMR (Structured Adaptive Mesh Refinement) simulations. 
In this paper, the numerical solution of Huxley equation by using two finite difference methods and stability analysis of these two methods are analyzed.

\section{The Mathematical Model}

One of the famous non-linear reaction-diffusion equations is the generalized Burgers-Huxley ( $\mathrm{gBH})$ equation:

$$
\begin{aligned}
& \frac{\partial u}{\partial t}+\alpha u^{\delta} \frac{\partial u}{\partial x}-\frac{\partial^{2} u}{\partial x^{2}}=\beta u\left(1-u^{\delta}\right)\left(u^{\delta}-a\right) \\
& \alpha \geq 0, \beta \geq 0, \delta>0, \text { and } \mathrm{a} \in(0,1)
\end{aligned}
$$

If we take $\delta=1, \alpha \neq 0$, and $\beta \neq 0$, equation (1) becomes the following Burgers-Huxley $(\mathrm{BH})$ equation:

$$
\frac{\partial u}{\partial t}+\alpha u \frac{\partial u}{\partial x}-\frac{\partial^{2} u}{\partial x^{2}}=\beta u(1-u)(u-a)
$$

Equation (2) shows a prototype model for describing the interaction mechanism, convection transport. When $\beta=0$, and $\delta=1$, equation (1) is reduced to Burgers equation which describes the far field of wave propagation in nonlinear dissipative systems

$\frac{\partial u}{\partial t}+\alpha u \frac{\partial u}{\partial x}-\frac{\partial^{2} u}{\partial x^{2}}=0$

When $\alpha=0$, and $\delta=1$, equation (1) is reduced to the Huxley equation which describes nerve pulse propagation in nerve fibers and wall motion in liquid crystal

$$
\frac{\partial u}{\partial t}-\frac{\partial^{2} u}{\partial x^{2}}=\beta u(1-u)(u-a)
$$

It is known that nonlinear diffusion equations (3) and (4) play important roles in nonlinear physics. They are of special significance for studying nonlinear phenomena [19]. Zeldovich and Frank- Kamenetsky formulated the equation (4) in 1938 as a model for flame front propagation and for this reason this equation sometimes named Zeldovich-FrankKamenetsky (ZF) equation, which has been extensively studied as a simple nerve model [1]. In 1952 Hodgkin and Huxley proposed their famous Hodgkin-Huxley model for nerve propagation. Because of the mathematical complexity of this model, it led to the introduction of the simpler FitzhughNagumo system. The simplified model of the Fitzhugh-Nagumo system is Huxley equation [18]. Because Huxley equation is a special case of Fitzhugh-Nagumo system, it is sometimes named Fitzhugh-Nagumo (FN) equation [5] or the reduced Nagumo equation or Nagumo equation [15]. In sixties, Fitzhugh used equation (4) as an approximate equation for the 
description of dynamics of the giant axon. This equation was among the first models of excited media [8].

In this paper, we shall take the Huxley equation as a model problem [12]:

$\frac{\partial u}{\partial t}-\frac{\partial^{2} u}{\partial x^{2}}=\beta u(1-u)(u-a)$

$\mathrm{x} \in[-\mathrm{L}, \mathrm{L}], t \geq 0$

$u(x, 0)=(b-H) x^{2}+H, b \geq 0, H>0$

$u(-L, t)=u(L, t)=b$

For the purpose of numerical calculations, we shall take:

$\beta=1, a \in(0,1), L=1,0 \leq b \leq 1$, and $0<H \leq 1,0 \leq t \leq 3$.

3. Derivation of the Explicit Scheme Formula of Huxley Equation [14] is $R=\{(x, t):-L \leq x \leq L, 0 \leq t \leq c\} \quad$ Assume that the rectangle subdivided into $(n-1)$ by $(m-1)$ rectangles with sides $\Delta x=h, \Delta t=k$.

Start at the bottom row, where $t=t_{1}=0$, and the initial condition is [12]:

$u\left(x_{i}, t_{1}\right)=f\left(x_{i}\right)=(b-H) x_{i}^{2}+H, i=2,3, \ldots, n-1$.

A method for computing the approximations to $u(x, t)$ at grid points in successive rows will be developed

$\left\{u\left(x_{i}, t_{j}\right): i=2,3,4, \ldots, n-1\right\}, j=2,3,4, \ldots, m$

The difference formulas used for $u_{t}(x, t)$ and $u_{x x}(x, t)$ are:

$u_{t}(x, t)=\frac{u(x, t+k)-u(x, t)}{k}+O(k)$

$u_{x x}(x, t)=\frac{u(x+h, t)-2 u(x, t)+u(x-h, t)}{h^{2}}+O\left(h^{2}\right)$

Where the grid points are:

$x_{i+1}=x_{i}+h, x_{i-1}=x_{i}-h, t_{j+1}=t_{j}+k, t_{j-1}=t_{j}-k$

Neglecting the terms $\mathrm{O}(k)$ and $\mathrm{O}\left(h^{2}\right)$, and use approximation $u_{i, j}$ for $u\left(x_{i}, t_{j}\right)$ in equations (8) and (9), which are in turn substituted in equation (4), we get

$\frac{\mathrm{u}_{\mathrm{i}, \mathrm{j}+1}-u_{i, j}}{k}-\frac{u_{i+1, j}-2 u_{i, j}+u_{i-1, j}}{h^{2}}=\beta u_{i, j}\left(1-u_{i, j}\right)\left(u_{i, j}-a\right)$

From equation (10), we have

$u_{i, j+1}-u_{i, j}=r\left(u_{i+1, j}-2 u_{i, j}+u_{i-1, j}\right)+k \beta u_{i, j}\left(1-u_{i, j}\right)\left(u_{i, j}-a\right)$

Where $r=k / h^{2}$ 
After some mathematical manipulation, we obtain

$u_{i, j+1}=r\left(u_{i-1, j}+u_{i+1, j}\right)+(1-2 r-k \beta a) u_{i, j}+k \beta\left(u_{i, j}\right)^{2}\left(1+a-u_{i, j}\right)$

Equation (12) represents the explicit finite difference formula for equation (4). Equation (12) is employed to create $(j+1)$ th row across the grid, assuming that approximations in the $j$ th row are known.

Notice that this formula explicitly gives the value $u_{i, j+1}$ in terms of $u_{i-1, j}, u_{i, j}$, and $u_{i+1, j}$.

4. Stability Analysis of the Explicit Scheme Using Fourier (von Neumann) Method

The basic idea of this method is to replace the solution of the finite difference method $u_{n, m}$ at time $t$ by $\psi(t) e^{i \gamma x}$, where $i=\sqrt{-1}, \gamma>0$ [16]. To apply von Neumann method to equation (4), we resort to the linearized stability analysis [7], we have

$\frac{\partial u}{\partial t}=\frac{\partial^{2} u}{\partial x^{2}}-a \beta u$

The finite difference explicit formula for (13) is:

$\frac{u_{n, m+1}-u_{n, m}}{\Delta t}=\frac{u_{n+1, m}-2 u_{n, m}+u_{n-1, m}}{(\Delta x)^{2}}-a \beta u_{n, m}$

Substituting $u_{n, m}=\psi(t) e^{i \gamma x}$ in (14), we have

$\frac{\psi(t+\Delta t) e^{i \gamma x}-\psi(t) e^{i \gamma x}}{\Delta t}=\frac{\psi(t) e^{i \gamma(x+\Delta x)}-2 \psi(t) e^{i \gamma x}+\psi(t) e^{i \gamma(x-\Delta x)}}{(\Delta x)^{2}}-a \beta \psi(t) e^{i \gamma x} \Rightarrow$

$\left[\frac{\psi(t+\Delta t)-\psi(t)}{\Delta t}\right] e^{i \gamma x}=\frac{\psi(t)}{(\Delta x)^{2}}\left[e^{i \gamma \Delta x}+e^{-i \gamma \Delta x}-2\right] e^{i \gamma x}-a \beta \psi(t) e^{i \gamma x} \Rightarrow$

$\psi(t+\Delta t)-\psi(t)=r \psi(t)\left[e^{i \gamma \Delta x}+e^{-i \gamma \Delta x}-2\right]-a \beta \Delta t \psi(t)$

Where $r=k / h^{2}$ 


$$
\begin{aligned}
& \psi(t+\Delta t)-\psi(t)=r \psi(t)[\cos \gamma \Delta x+i \sin \gamma \Delta x+\cos \gamma \Delta x-i \sin \gamma \Delta x-2]-a \beta \Delta t \psi(t) \\
& =r \psi(t)[2 \cos \gamma \Delta x-2]-a \beta \Delta t \psi(t) \\
& =2 r \psi(t)[\cos \gamma \Delta x-1]-a \beta \Delta t \psi(t) \\
& =-2 r \psi(t)[1-\cos \gamma \Delta x]-a \beta \Delta t \psi(t) \\
& =-2 r \psi(t)\left[1-\left(1-2 \sin ^{2}(\gamma \Delta x / 2)\right)\right]-a \beta \Delta t \psi(t) \\
& =-4 r \psi(t) \sin ^{2}(\gamma \Delta x / 2)-a \beta \Delta t \psi(t) \Rightarrow \\
& \psi(t+\Delta t)=\psi(t)-4 r \psi(t) \sin ^{2}(\gamma \Delta x / 2)-a \beta \Delta t \psi(t) \\
& =\left[1-4 r \sin ^{2}(\gamma \Delta x / 2)-a \beta \Delta t\right] \psi(t) \Rightarrow \\
& \psi(t+\Delta t) / \psi(t)=1-4 r \sin ^{2}(\gamma \Delta x / 2)-a \beta \Delta t=\xi
\end{aligned}
$$

Where $\xi$ can be visualized as the amplification factor and we get

$$
\psi(t+\Delta t) / \psi(t)=\xi
$$

As we advance the solution from a particular plane $\psi(t)$ to the next plane $\psi(t+\Delta t),|\psi(t+\Delta t)-\psi(t)|$ must start decreasing or alternatively $\psi(t)$ must be bounded function, i.e. $\psi(t)$ should not tend infinity for large $t$.

From equation (15), for boundedness of (15), we need

$$
\begin{aligned}
& |\psi(t+1) / \psi(t)| \leq 1 \Rightarrow \\
& |\xi| \leq 1 \Rightarrow \\
& \left|1-4 r \sin ^{2}(\gamma \Delta x / 2)-a \beta \Delta t\right| \leq 1
\end{aligned}
$$

In the above inequality, the right-side inequality is:

$$
1-4 r \sin ^{2}(\gamma \Delta x / 2)-a \beta \Delta t \leq 1
$$

Implies $r>0$ and this is always true.

Hence, in order that (16) is to be satisfied, we need

$-1 \leq 1-4 r \sin ^{2}(\gamma \Delta x / 2)-a \beta \Delta t \Rightarrow$

$-2 \leq-4 r \sin ^{2}(\gamma \Delta x / 2)-a \beta \Delta t \Rightarrow$

$2 \geq 4 r \sin ^{2}(\gamma \Delta x / 2)+a \beta \Delta t \Rightarrow$

$\frac{1}{2}-\frac{a \beta \Delta t}{4} \geq r \sin ^{2}(\gamma \Delta x / 2)$

For some $\beta, \sin ^{2}(\gamma \Delta x / 2)$ is unity and hence the above condition reduces to 
$r \leq \frac{2-a \beta \Delta t}{4}$

Since $r=\Delta t /(\Delta x)^{2}$, from inequality (17), we have

$\Delta t \leq \frac{2(\Delta x)^{2}}{4+a \beta(\Delta x)^{2}}$ stable.

This precisely the conditions imposed on the explicit scheme to be

\section{Derivation of the Crank-Nicholson Scheme Formula of Huxley Equation}

The is diffusion term $u_{x x}$ in this method is represented by central differences, with their values at the current and previous time steps averaged [17]:

$$
\begin{aligned}
& u_{x x}=\frac{1}{2}\left[\left(\frac{u(x-h, t+k)-2 u(x, t+k)+u(x+h, t+k)+u(x-h, t)-2 u(x, t)+u(x+h, t)}{h^{2}}\right)\right] \\
& \mathrm{u}_{\mathrm{t}}=\frac{u(x, t+k)-u(x, t)}{k}
\end{aligned}
$$

By using the approximation $u_{i, j}$ for $u\left(x_{i}, t_{j}\right)$ in equations (19) and (20), which are in turn substituted into equation (4), we have

$$
\frac{u_{i, j+1}-u_{i, j}}{k}-\frac{u_{i+1, j+1}-2 u_{i, j+1}+u_{i-1, j+1}}{2 h^{2}}-\frac{u_{i+1, j}-2 u_{i, j}+u_{i-1, j}}{2 h^{2}}=\beta u_{i, j}\left(1-u_{i, j}\right)\left(u_{i, j}-a\right)
$$

From (21), we get

$2 u_{i, j+1}-2 u_{i, j}-r\left(u_{i+1, j+1}-2 u_{i, j+1}+u_{i-1, j+1}\right)$

$=r\left(u_{i+1, j}-2 u_{i, j}+u_{i-1, j}\right)+2 k \beta\left(u_{i, j}-\left(u_{i, j}\right)^{2}\right)\left(u_{i, j}-a\right)$

$r=k / h^{2}$ Where

After some mathematical manipulation, we get

$-r\left(u_{i-1, j+1}+u_{i+1, j+1}\right)+(2+2 r) u_{i, j+1}=r\left(u_{i-1, j}+u_{i+1, j}\right)+(2-2 r-2 a k \beta) u_{i, j}$

$+2 k \beta\left(u_{i, j}\right)^{2}\left(1+a-u_{i, j}\right), \quad i=2,3,4, \ldots, n-1$

Equation (22) represents the Crank-Nicholson formula for equation (4).

The terms on the right-hand side of equation (22) are all known. Hence, the equations in (22) form a tridiagonal linear algebraic system $A X=B$.

The boundary conditions are used in the first and last equations only i.e. $u_{1, j}=u_{1, j+1}=b$, and $u_{n, j}=u_{n, j+1}=b, \forall j$. 
Equations in (22) are especially pleasing to view in their tridiagonal matrix form $A X=B$, where $\mathrm{A}$ is the coefficient matrix, $\mathrm{X}$ is the unknown vector and $\mathrm{B}$ is the known vector as shown below:

$$
\begin{aligned}
& {\left[\begin{array}{ccccc}
2+2 r & -r & & & \\
-r & 2+2 r & -r & & \\
& & & & \\
& & 2+2 r & -r & \\
& & & 2+2 r & -r \\
& & & -r & 2+2 r
\end{array}\right]\left[\begin{array}{c}
u_{2, j+1} \\
u_{3, j+1} \\
u_{p, j+1} \\
\vdots \\
\mathrm{u}_{\mathrm{n}-2, \mathrm{j}+1} \\
\mathrm{u}_{\mathrm{n}-1, \mathrm{j}+1}
\end{array}\right]=} \\
& {\left[\begin{array}{c}
2 r b+(2-2 r-2 a \beta k) u_{2, j}+2 \beta k\left(u_{2, j}\right)^{2}\left(1+a-u_{2, j}\right)+r u_{3, j} \\
r\left(u_{2, j}+u_{4, j}\right)+(2-2 r-2 a \beta k) u_{3, j}+2 \beta k\left(u_{3, j}\right)^{2}\left(1+a-u_{3, j}\right) \\
: \\
\mathrm{r}\left(u_{p-1, j}+u_{p+1, j}\right)+(2-2 r-2 a \beta k) u_{p, j}+2 \beta k\left(u_{p, j}\right)^{2}\left(1+a-u_{p, j}\right) \\
: \\
\mathrm{r}\left(u_{n-3, j}+u_{n-1, j}\right)+(2-2 r-2 a \beta k) u_{n-2, j}+2 \beta k\left(u_{n-2, j}\right)^{2}\left(1+a-u_{n-2, j}\right) \\
r u_{n-2, j}+(2-2 r-2 a \beta k) u_{n-1, j}+2 r b
\end{array}\right]}
\end{aligned}
$$

When the Crank-Nicholson scheme is implemented with a computer, the linear system $A X=B$ can be solved by either direct means or by iteration. In this paper, the Gaussian elimination method (direct method) has been used to solve the algebraic system $\mathrm{AX}=\mathrm{B}$.

\section{Stability Analysis of the Crank- Nicholson Scheme Using Fourier (von Neumann) Method}

The finite difference Crank-Nicholson formula for (13) is:

$\frac{u_{n, m+1}-u_{n, m}}{\Delta t}=\frac{u_{n+1, m+1}-2 u_{n, m+1}+u_{n-1, m+1}}{2(\Delta x)^{2}}+\frac{u_{n+1, m}-2 u_{n, m}+u_{n-1, m}}{2(\Delta x)^{2}}-a \beta \mathrm{u}_{\mathrm{n}, \mathrm{m}}$

Substituting $u_{n, m}=\psi(t) e^{i \gamma x}$ in equation (23), we have 


$$
\begin{aligned}
& \frac{\psi(t+\Delta t) e^{i \gamma x}-\psi(t) e^{i j x}}{\Delta t}=\frac{\psi(t+\Delta t) e^{i \gamma(x+\Delta x)}-2 \psi(t+\Delta t) e^{i \gamma x}+\psi(t+\Delta t) e^{i \gamma(x-\Delta x)}}{2(\Delta x)^{2}}+ \\
& \frac{\psi(t) e^{i \gamma(x+\Delta x)}-2 \psi(t) e^{i j x}+\psi(t) e^{i \gamma(x-\Delta x)}}{2(\Delta x)^{2}}-a \beta \psi(t) e^{i \gamma x} \Rightarrow \\
& {\left[\frac{\psi(t+\Delta t)-\psi(t)}{\Delta t}\right] e^{i \gamma x}=\frac{\psi(t+\Delta t)}{2(\Delta x)^{2}}\left[e^{i \gamma \Delta x}+e^{-i \gamma \Delta x}-2\right] e^{i \gamma x}+\frac{\psi(t)}{2(\Delta x)^{2}}\left[e^{i \gamma \Delta x}+e^{-i \gamma \Delta x}-2\right] e^{i \gamma x}-} \\
& a \beta \psi(t) e^{i \gamma x} \Rightarrow \\
& \psi(t+\Delta t)-\psi(t)=\frac{r \psi(t+\Delta t)}{2}\left[e^{i \gamma \Delta x}+e^{-i \gamma \Delta x}-2\right]+\frac{r \psi(t)}{2}\left[e^{i \gamma \Delta x}+e^{-i \gamma \Delta x}-2\right]-a \beta \Delta t \psi(t)
\end{aligned}
$$

Where $r=k / h^{2}$

$$
\begin{aligned}
& \psi(t+\Delta t)-\psi(t)=\frac{r \psi(t+\Delta t)}{2}[\cos \gamma \Delta x+i \sin \gamma \Delta x+\cos \gamma \Delta x-i \sin \gamma \Delta x-2] \\
& +\frac{r \psi(t)}{2}[\cos \gamma \Delta x+i \sin \gamma \Delta x+\cos \gamma \Delta x-i \sin \gamma \Delta x-2]-a \beta \Delta t \psi(t) \\
& =\frac{r \psi(t+\Delta t)}{2}[2 \cos \gamma \Delta x-2]+\frac{r \psi(t)}{2}[2 \cos \gamma \Delta x-2]-a \beta \Delta t \psi(t) \\
& =r \psi(t+\Delta t)[\cos \gamma \Delta x-1]+r \psi(t)[\cos \gamma \Delta x-1]-a \beta \Delta t \psi(t) \\
& =-r \psi(t+\Delta t)[1-\cos \gamma \Delta x]-r \psi(t)[1-\cos \gamma \Delta x]-a \beta \Delta t \psi(t) \\
& =-r \psi(t+\Delta t)\left[1-\left(1-2 \sin ^{2}(\gamma \Delta x / 2)\right)\right]-r \psi(t)\left[1-\left(1-2 \sin ^{2}(\gamma \Delta x / 2)\right)\right]-a \beta \Delta t \psi(t) \\
& =-2 r \psi(t+\Delta t) \sin ^{2}(\gamma \Delta x / 2)-2 r \psi(t) \sin ^{2}(\gamma \Delta x / 2)-a \beta \Delta t \psi(t) \Rightarrow \\
& \psi(t+\Delta t)+2 r \psi(t+\Delta t) \sin ^{2}(\gamma \Delta x / 2)=\psi(t)-2 r \psi(t) \sin ^{2}(\gamma \Delta x / 2)-a \beta \Delta t \psi(t) \Rightarrow \\
& \left(1+2 r \sin ^{2}(\gamma \Delta x / 2)\right) \psi(t+\Delta t)=\left(1-2 r \sin ^{2}(\gamma \Delta x / 2)-a \beta \Delta t\right) \psi(t) \Rightarrow \\
& \psi(t+\Delta t) / \psi(t)=\frac{1-2 r \sin ^{2}(\gamma \Delta x / 2)-a \beta \Delta t}{1+2 r \sin ^{2}(\gamma \Delta x / 2)} \Rightarrow \\
& \psi(t+\Delta t) / \psi(t)=\frac{1-\left[2 r \sin ^{2}(\gamma \Delta x / 2)+a \beta \Delta t\right]}{1+2 r \sin ^{2}(\gamma \Delta x / 2)}=\xi \Rightarrow \psi(t+\Delta t) / \psi(t)=\xi
\end{aligned}
$$

For stability, we need

$$
\begin{aligned}
& |\psi(t+\Delta t) / \psi(t)| \leq 1 \Rightarrow \\
& |\xi| \leq 1 \Rightarrow\left|\frac{1-\left[2 r \sin ^{2}(\gamma \Delta x / 2)+a \beta \Delta t\right]}{1+2 r \sin ^{2}(\gamma \Delta x / 2)}\right| \leq 1, \forall r, a, \beta, \Delta t
\end{aligned}
$$

Hence, the Crank-Nicholson scheme is unconditionally stable.

\section{Conclusions}

We concluded from the comparison between the two schemes that the explicit scheme is easier and has faster convergence than the Crank- 
Nicholson scheme which is more accurate than the explicit scheme and the results of this paper are affirming the analytical results which obtained by Manoranjan et al [12] as shown below:

$$
\begin{gathered}
\text { (1) If } b=0 \text { then } . u(x, t) \rightarrow 0 \text { as } t \rightarrow \infty \text { if } L<\pi /(1-a) \\
. u(x, t) \rightarrow a \text { as } t \rightarrow \infty \text { if } L<\pi \text { (2) If } b=a \text { then } \\
. u(x, t) \rightarrow 1 \text { as } t \rightarrow \infty \text { if } L<\pi / a \text { (3) If } b=1 \text { then }
\end{gathered}
$$

as shown in figure (1) and table (1). In addition, from stability analysis, we concluded that the explicit scheme is conditionally stable if $r \leq \frac{2-a \beta \Delta t}{4}, \Delta t \leq \frac{2(\Delta x)^{2}}{4-a \beta(\Delta x)^{2}}$ while the Crank-Nicholson scheme is unconditionally stable.

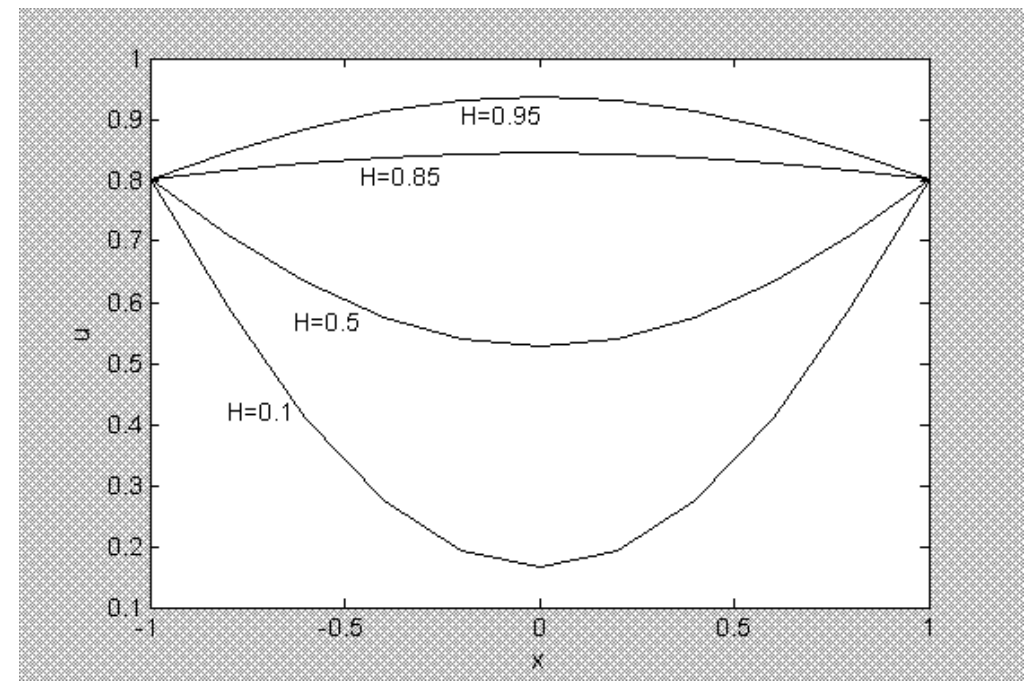

Figure (1)

Figure (1) Explains the solution of the Huxley equation by the use of Crank-Nicholson scheme for various values of $\mathrm{H}$ at $\mathrm{a}=\mathrm{b}=0.8$.

The figure shows that the solution of the problem converges to the steady state solution $\mathrm{u}=\mathrm{a}=0.8$ as $\mathrm{t}$ gets large at specific boundary condition $\mathrm{b}=$ 0.8 . 
Table (1)

\begin{tabular}{|c|c|c|c|}
\hline $\begin{array}{c}\text { Explicit } \\
\mathrm{b}=0.25, \mathrm{a}=0.25, \\
\mathrm{H}=0.1\end{array}$ & $\begin{array}{c}\text { Crank-Nicholson } \\
\mathrm{b}=0.25, \mathrm{a}=0.25, \\
\mathrm{H}=0.1\end{array}$ & $\begin{array}{c}\text { Explicit } \\
\mathrm{b}=0.25, \\
\mathrm{a}=0.25, \mathrm{H}=0.3\end{array}$ & $\begin{array}{c}\text { Crank-Nicholson } \\
\mathrm{b}=0.25, \mathrm{a}=0.25, \\
\mathrm{H}=0.3\end{array}$ \\
\hline 0.1000 & 0.1000 & 0.3000 & 0.3000 \\
\hline 0.1287 & 0.1274 & 0.2910 & 0.2914 \\
\hline 0.1573 & 0.1513 & 0.2817 & 0.2836 \\
\hline 0.1771 & 0.1706 & 0.2751 & 0.2772 \\
\hline 0.1933 & 0.1862 & 0.2696 & 0.2720 \\
\hline 0.2056 & 0.1987 & 0.2654 & 0.2678 \\
\hline 0.2152 & 0.2087 & 0.2621 & 0.2644 \\
\hline 0.2228 & 0.2167 & 0.2595 & 0.2616 \\
\hline 0.2287 & 0.2232 & 0.2575 & 0.2594 \\
\hline 0.2333 & 0.2284 & 0.2559 & 0.2576 \\
\hline 0.2369 & 0.2326 & 0.2546 & 0.2561 \\
\hline 0.2397 & 0.2359 & 0.2536 & 0.2549 \\
\hline 0.2420 & 0.2387 & 0.2528 & 0.2540 \\
\hline 0.2437 & 0.2409 & 0.2522 & 0.2532 \\
\hline 0.2451 & 0.2426 & 0.2517 & 0.2526 \\
\hline 0.2461 & 0.2440 & 0.2514 & 0.2521 \\
\hline 0.2470 & 0.2452 & 0.2511 & 0.2517 \\
\hline 0.2476 & 0.2461 & 0.2508 & 0.2514 \\
\hline
\end{tabular}

Table (1) shows the solution of Huxley equation by the use of Crank-Nicholson scheme and explicit scheme for some values of $a, b$, and $\mathrm{H}$

The table above explains that the solution of the two schemes converges to the steady state solution $\mathrm{u}=\mathrm{a}=0.25$ and the number of steps which are needed to reach the solution $\mathrm{u}=\mathrm{a}=0.25$ in the explicit scheme is less than the number of steps in the Crank-Nicholson scheme at specific boundary condition $\mathrm{b}=0.25$ and $\mathrm{H}=0.1,0.3$.

\section{Acknowledgement}

The authors would like to express their gratitude and indebtedness to every person helped in this paper. 


\section{REFERENCES}

[1] Binczak, S., Eilbeck, J.C. and Scott, A.C., (2001), Ephaptic Coupling of Myelinated Nerve Fibers, Physica D 148 pp. 159-174.

[2] Broadbridge, P., Bradshaw, B.H., Fulford, G.R. and Aldis, G.K., (2002), Huxley and Fisher Equations for Gene Propagation: An Exact Solution, ANZIAM J. Vol. 44, part 1, pp. 11-20.

[3] Eilbeck, J.C. and Manoranjan, V.S., (1986), A Comparison of Basis Functions for The Pseudo-Spectral Method for a Model Reaction-Diffusion Problem, J. Comput. Appl. Math., 15, pp. 371378.

[4] Eilbeck, J. C., (1986), The Pseudo-Spectral Method and Path Following in Reaction-Diffusion Bifurcation Studies, SIAM J. Sci. Stat. Comput., Vol. 7, No. 2, pp. 599-610.

[5] Estevez, P.G. and Gordoa, P.R., (1990), Painleve Analysis of the Generalized Burgers-Huxley Equation, J. Phys. A: Math. Gen., 23, pp. 4831-4837.

[6] Fath, G. and Domanski, Z., (1999), Avalanche of Bifurcations and Hysteresis in a Model of Cellular Differentiation, Phys. Rev. E. Vol. 60, No. 4, pp. 4604-4609.

[7] Garbey, M., Kaper, H.G., and Romanyukha, N., (2001), A Fast Solver for Systems of Reaction-Diffusion Equations, Thirteenth International Conference on Domain Decomposition Methods, Editors: Debit, N., Garbey, M., Hoppe, R., Periaus, J., Keses, D. and Knznetsov, Y., pp. 385-392.

[8] Landa, P.S., (1996), Nonlinear Oscillations and Waves in Dynamical Systems, Kluwer Academic Publishers.

[9] Lefantzi, S., Kennedy, C.A., Ray, J. and Najm, H. N., (2003), A Study of the Effect of Higher Order Spatial Discretizations in SAMR (Structured Adaptive Mesh Refinement) Simulations, Proceedings of the Fall Meeting of the Western States Section of The Combustion Institute, Los Angeles, AC.

[10] Lewis, T. J. and Keener, J.P., (2000), Wave-Block in Excitable Media Due to Regions of Depressed Excitability, SIAM J. Appl. Math., Vol. 61, No.1, pp. 293-316.

[11] Logan, J.D., (1987), Applied Mathematics, John Wiley \& Sons. 
[12] Manoranjan, V.S., Mitchell, A.R., Sleeman, B.D. and Yu, K. P., (1984), Bifurcation Studies in Reaction-Diffusion, J. Comput. Appl. Math., 11, pp. 27-37.

[13] Manoranjan, V.S., (1984), Bifurcation Studies in ReactionDiffusion II, J. Comput. Appl. Math., 11, pp. 307-314.

[14] Mathews, J.H. and Fink, K.D., (1999), Numerical Methods Using MATLAB, Prentice-Hall, Inc.

[15] Pesin, Y. and Yurchenko, A., (2004), Some Physical Models of the Reaction-Diffusion Equation and Coupled Map Lattices, Russian Math. Surveys, Vol. 59, No. 3, to appear.

[16] Shanthakumar, M., (1989), Computer Based Numerical Analysis, Khanna Publishers.

[17] Sherratt, J. A., (1996), Oscillatory and Chaotic Wakes Behind Moving Boundaries in Reaction-Diffusion Systems, J. Dynamics and Stability of Systems, Vol. 11, No. 4, pp. 303-324.

[18] Wang, X.Y., (1985), Nerve Propagation and Wall in Liquid Crystals, Phys. Lett., Vol. 112 A, No. 8, pp. 402-406.

[19] Wang, X. Y., Zhu, Z. S. and Lu, Y. K., (1990), Solitary Wave Solutions of the Generalized Burgers-Huxley Equation, J. Phys. A: Math. Gen., 23, pp. 271-274. 